\title{
REALIZATION OF LONG EXACT SEQUENCES OF ABELIAN GROUPS
}

\author{
IRWIN S. PRESSMAN
}

\begin{abstract}
Given a long exact sequence of abelian groups

L :

$\ldots \longrightarrow L^{i-1} \stackrel{\xi^{i-1}}{\longrightarrow} L^{i} \stackrel{\xi^{i}}{\longrightarrow} L^{i+1} \longrightarrow \ldots$

a short exact sequence of complexes of free abelian groups is constructed whose cohomology long exact sequence is precisely $L$. In this sense, L is realized. Two techniques which are introduced to reduce or replace lengthy diagram chasing arguments may be of interest to some readers. One is an arithmetic of bicartesian squares; the other is the use of the fact that categories of morphisms of abelian categories are themselves abelian.
\end{abstract}

\section{0 . Introduction}

There are few objects in Algebraic Topology or Homological Algebra which are more familiar than long exact sequences, nor techniques which are more common than diagram chasing. This paper is directed towards first proving that every long exact sequence is in fact the cohomology sequence of a short exact sequence of complexes of abelian groups, and then demonstrating that these groups can all be chosen to be free. In the proof of these results, it is shown how bicartesian squares can be efficiently utilized to compute cohomology groups and prove exactness, without appealing to diagram chasing and long lists of names of functions and variables. An arithmetic of bicartesian squares is developed and used.

Later, in the proof that the complexes can be free, standard facts about abelian categories are used to verify the existence of a commutative diagram of complexes (3.9), where the usual proofs of commutativity would have needed complicated 4-dimensional diagram chasing. Hence it is possible that the methods used here may be of interest to some readers, for their own sake. 


\section{Definitions and Notation}

Unless it is stated otherwise, all objects and morphisms will be abelian groups and group homomorphisms from the category $\mathbf{A} \mathbf{b}$ of abelian groups. All groups will be denoted by English capital letters: $A, B, C, \ldots A$ complex $\mathcal{B}$ is a doubly infinite sequence of abelian groups

$B$ :

$$
\ldots \longrightarrow B^{m-1} \stackrel{d^{m-1}}{\longrightarrow} B^{m} \stackrel{d^{m}}{\longrightarrow} B^{m+1} \longrightarrow \ldots
$$

such that $d^{m} d^{m-1}=0$ for all $m \in \mathbf{Z}$. Complexes will be denoted by script letters, and their $k$ th group $B^{k}$ and $k$ th homomorphism $d^{k}$ will be denoted by $[B]^{k}$ and $[\delta]^{k}$.

If the complex $\mathcal{B}$ is viewed as shifted one position to the left, and if the signs of each homomorphism in the complex are reversed, then the new complex will be denoted by $\Sigma \mathcal{B}$. where $[\Sigma \mathcal{B}]^{k}=B^{k+1}$ and $[\Sigma \delta]^{k}=-d^{k+1}$. If the category of all complexes of abelian groups, and all degree preserving homomorphisms $h: \mathcal{B} \longrightarrow \mathcal{C}$ of complexes

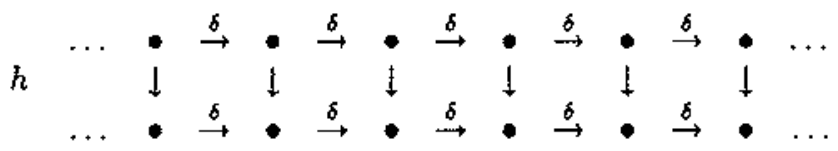

is denoted by $\mathcal{C} A B$, then $\Sigma: \mathcal{C A B} \longrightarrow \mathcal{C A B}$ is a functor. $\mathcal{C A B}$ is an abelian category [2,3]. Moreover, the category of morphisms of $\mathcal{C} A \mathcal{B}, \mathcal{C A B}{ }^{2}$, whose objects are homomorphisms $\left(\begin{array}{l}a \\ b\end{array}\right): g \rightarrow h$ such that $h a=b g$ is a commutative diagram of complexes, is also abelian.

A bicartesian square (bcs) is a commutative diagram (1.1) which is both a pullback and a pushout.

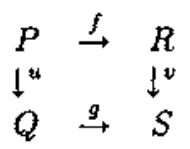

This is equivalent to stating that

$$
O \longrightarrow P \stackrel{\{u, f\}}{\rightarrow} Q \oplus R \stackrel{\langle g,-v\rangle}{\rightarrow} S \longrightarrow O
$$

is a short exact sequence (ses). This is called the ses of the bes (1.1). Note the standard usage of $\rightarrow$ to denote monomorphism, and $\rightarrow$ to denote epimorphism. Following the notation of [2], given $f_{1}: A_{1} \rightarrow B_{1}, f_{2}: A_{1} \rightarrow$ $B_{2}, g_{1}: A_{2} \rightarrow B_{1}$, and $g_{2}: A_{2} \rightarrow B_{2}$, then denote the canonical maps into coproducts of abelian groups (respectively from products) as $\left\{f_{1}, f_{2}\right\}: A_{1} \rightarrow$ $\left.B_{1} \oplus B_{2}\left(<f_{2}, g_{2}\right\rangle: A_{1} \oplus A_{2} \rightarrow B_{2}\right)$. The homomorphism $\left\{<f_{1}, g_{1}\right\rangle,<$ $\left.f_{2}, g_{2}>\right\}: A_{1} \oplus A_{2} \rightarrow B_{1} \oplus B_{2}$ will be denoted by $\left[f_{1}, g_{1}: f_{2}, g_{2}\right]$, which is, of course, equal to $<\left\{f_{1}, f_{2}\right\},\left\{g_{1}, g_{2}\right\}>$ by the commutative laws. 
In a bcs (1.1), opposite pairs of morphisms have the same kernel and same cokernel. Therefore, $u$ is a monomorphism iff $r$ is a monomorphism; $f$ is an epimorphism iff $g$ is an epimorphism. If two bcs's $\mathbf{C}$ and $\mathbf{D}$ are composed along a common edge $R \rightarrow S$ to form a new square $\mathbf{E}$, then the resulting square $\mathbf{E}$ is also bicartesian. Denote $\mathbf{E}$ by $[\mathbf{C}+\mathbf{D}]$.

$$
\begin{array}{ccccc}
P & \longrightarrow & R & \longrightarrow & T \\
\downarrow & \mathbf{C} & \downarrow & \mathbf{D} & \downarrow \\
Q & \longrightarrow & S & \longrightarrow & U
\end{array} \Longrightarrow \begin{array}{llll}
P & \overrightarrow{\mathbf{E}} & \downarrow \\
\stackrel{Q}{\longrightarrow} & \longrightarrow
\end{array}
$$

In the special case where $Q=O$, the zero group, then the ses of $\mathrm{C}$ is $O \rightarrow P \rightarrow R \rightarrow S \rightarrow O$ and the ses of $[\mathrm{C}+\mathrm{D}]$ is $O \rightarrow P \rightarrow T \rightarrow U \rightarrow O$. It is well known that for an epimorphism $u: U \rightarrow V$ and a monomorphism $v: V \mapsto W$, there is a bcs (1.2) with $u$ and $v$ as adjacent edges [3, Corollary $3.8\}$

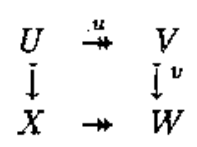

Call $X$ a South-West (SW) extension of $(u, v)$. In general, $X$ is not unique [1]. $X$ can be found precisely because the homological dimension of the category of abelian groups is 1 . This would fail in a category of modules over an arbitrary ring.

The standard $[2,3]$ facts about cohomology (or homology) of a ses of complexes are assumed here. Cohomology is used here, instead of homology, simply so that standard $\mathbb{Z}$ indexing can be adopted. The cohomology long exact sequence (les)

$$
\ldots \longrightarrow H^{n}(\mathcal{C}) \longrightarrow H^{n}(\mathcal{D}) \longrightarrow H^{n}(\mathcal{E}) \longrightarrow H^{n+1}(\mathcal{C}) \longrightarrow \ldots
$$

will be used frequently.

\section{The Construction of the Complexes}

In this section, one method of building a short exact sequence (ses) of complexes whose cohomology les is $\mathbf{L}$ is introduced. It is clear from the process that the ses of complexes is not unique. However, the methodology itself is of interest because it demonstrates the considerable advantages of using bicartesian squares instead of elements, as a basis of proof in diagram-chasing arguments. Assume that a les $\mathbf{L}$ is given.

$\mathbf{L}$ :

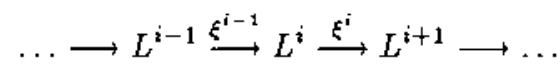


Since the les $\mathbf{L}$ is exact, $k e r \xi^{i}=i m \xi^{i-1}$. Denote $\operatorname{ker} \xi^{i}$ by $K^{i}$, so that for each $i$ there is a ses $K^{i} \nrightarrow L^{i} \rightarrow K^{i+1}$, and its corresponding bes

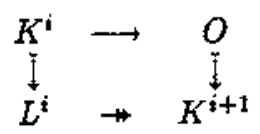

Use (1.2) to introduce new abelian groups $M^{i}$ by extending SW from $L^{i} \rightarrow$ $K^{i+1} \hookrightarrow L^{i+1}$ to obtain a bcs

$$
\underset{L^{i}}{\stackrel{1}{I}} \rightarrow \underset{M^{i}}{\rightarrow} K^{i+1}
$$

This SW-extension can be iterated, using $M^{i} \rightarrow L^{i+1} \mapsto M^{i+1}$ to obtain the bes

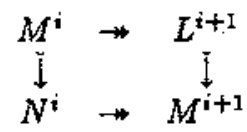

This process can be repeated as often as desired. These extensions glue together to create the commutative diagram of bcs's (2.1) which will be the main tool of this section.

$$
\begin{aligned}
& \text { (2.1) }
\end{aligned}
$$

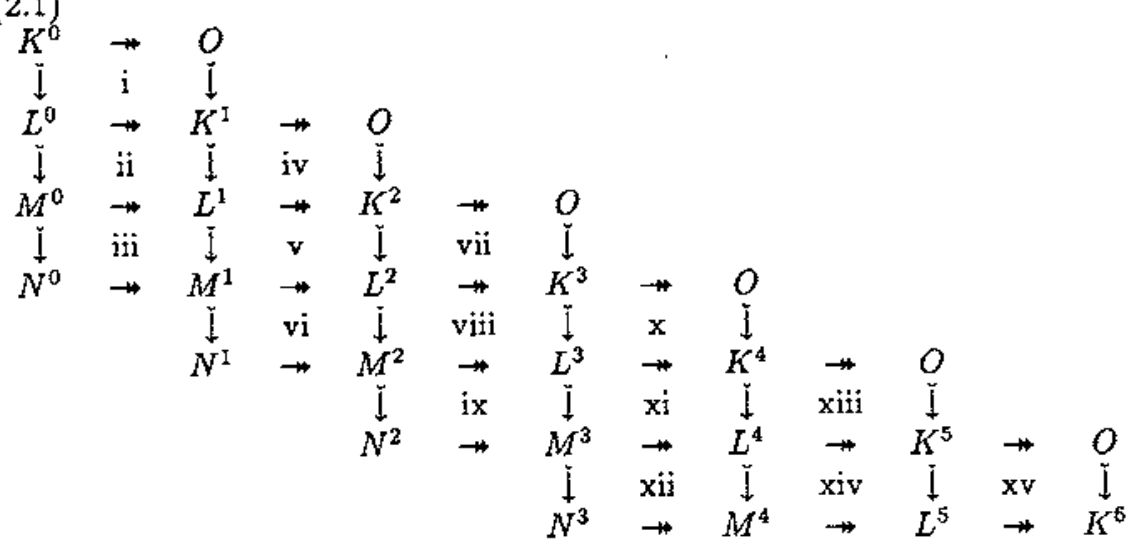

Consider the iterated compositions of squares $[i i+\mathrm{iv}]+[\mathrm{iii}+\mathrm{v}]$ and $[\mathrm{xi}+\mathrm{xiii}]$ $+[\mathrm{xii}+$ xiv $]$, e.g. $[\mathrm{xi}+$ xiii $]$ are composed along $K^{4} \longrightarrow L^{4}$, then [xii + xiv] along $L^{4} \longrightarrow M^{4}$ and then the two new bcs's are composed along $M^{3} \longrightarrow K^{5}$.

These produce two new bcs's

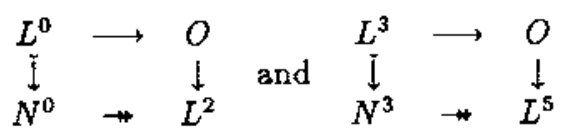


whose respective ses's are the third and fourth rows of the ses of vertical complexes (2.2).

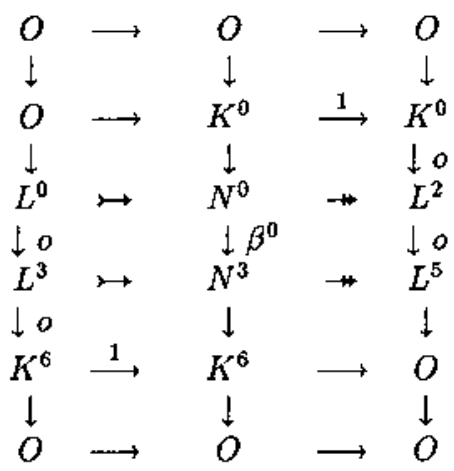

$\beta^{0}$ is defined to be the composition $N^{0} \rightarrow M^{1} \rightarrow L^{2} \rightarrow K^{3} \mapsto L^{3} \mapsto M^{3} \mapsto N^{3}$ of homomorphisms from (2.1). Clearly $K^{3}=i m \beta^{0}$. The ses of [iii $+\mathrm{v}+$ vii] is used to verify that $\operatorname{ker} \beta^{0}=\operatorname{ker}\left(N^{0} \rightarrow K^{3}\right)=M^{0}$.

Denote the cohomology group at the $i$ th row and $j$ th column of (2.2) by $H^{i, j}$, and the induced homomorphisms of cohomology by:

$$
\kappa^{i}: H^{i, 1} \longrightarrow H^{i, 2} \quad \lambda^{i}: H^{i, 2} \longrightarrow H^{i, 3} \quad \mu^{i}: H^{i, 3} \longrightarrow H^{i+1,1}
$$

The Lemma below is a crucial step in demonstrating that $\mathbf{L}$ can be realized as the cohomology les of a ses of complexes. The proof is given in detail. The bes's of (2.1) are used to verify that the cohomology groups of (2.2) and the induced homomorphisms agree with those of $\mathbf{L}$.

Lemma 2.3. The cohomology exact sequence of (2.2) is

$$
O \rightarrow O \rightarrow K^{0} \rightarrow L^{0} \stackrel{\xi^{0}}{\rightarrow} L^{1} \stackrel{\xi^{2}}{\rightarrow} L^{2} \stackrel{\xi^{2}}{\rightarrow} L^{3} \stackrel{\xi^{3}}{\rightarrow} L^{4} \stackrel{\xi^{4}}{\rightarrow} L^{5} \rightarrow K^{6} \rightarrow O \rightarrow O
$$

Proof: The vertical homomorphisms of columns 1 and 3 are all zero so the cohomology groups are equal to the group at each location. In column 2, $H^{1,2}=H^{2,2}=H^{5,2}=H^{6,2}=O$. The group $H^{3,2}=\left(\right.$ ker $\left.\beta^{0}\right) / K^{0}=M^{0} / K^{0}=$ $L^{1}$ where the last equality follows from the ses of the bcs [i + ii]. From [xii $+\mathrm{xiv}+\mathrm{xv}], M^{3}=\operatorname{ker}\left(N^{3} \longrightarrow K^{6}\right)$, so $H^{3,3}=M^{3} / i m \beta^{0}=M^{3} / K^{3}=L^{4}$, where the last equality follows from $[\mathrm{x}+\mathrm{xi}]$.

It is now verified that the induced homomorphisms $\kappa^{i}, \lambda^{i}, \mu^{i}$ are correct. The connecting homomorphism $\nu^{2}: H^{2,3} \longrightarrow H^{3,1}$ is induced by $K^{0} \longrightarrow N^{0}$ which factors through $L^{0}$ in the diagram of ses's arising from the bcs \#=[ii + iii]. This confirms that $\nu^{2}: K^{0} \longrightarrow L^{0}$ is the expected homomorphism.

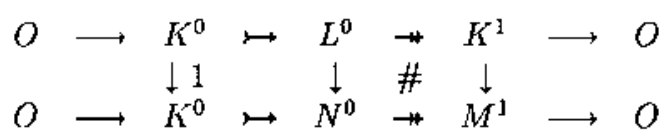


$\kappa^{3}$ is induced by the homomorphism of $L^{0}\left(=k e r\right.$ o: $\left.L^{0} \longrightarrow L^{3}\right)$ into $M^{0}=$ ker $\beta^{0}$ that arises from the given homomorphism $L^{0} \rightarrow N^{0}$ of $(2.2)$, which actually factors through $M^{0}$ by (2.1). This verifies that the induced map of cohomology groups, $\kappa^{3}: H^{3,1} \longrightarrow H^{3,2}$, becomes $L^{0} \longrightarrow M^{0} \longrightarrow M^{0} / K^{0}=$ $L^{1}$.

$\lambda^{3}: L^{1} \longrightarrow L^{2}$ is induced by $M^{0} \longrightarrow N^{0} \longrightarrow L^{2}$ which, by commutativity of (2.1), factors as $M^{0} \rightarrow L^{1} \rightarrow K^{2} \longrightarrow L^{2}$. Factoring out $K^{0}$ (the image) gives the induced map of cohomology $\lambda^{3}=\xi^{1}: L^{1} \longrightarrow L^{2}$.

The connecting homomorphism $\mu^{3}: H^{3,3} \longrightarrow H^{4,1}$, or $L^{2} \longrightarrow L^{3}$ is constructed by selecting any element of $L^{2}=$ ker $o: L^{2} \longrightarrow L^{5}$, lifting it to $N^{0}$, and mapping it to $N^{3}$ with $\beta^{0}$. However, since by definition $\beta^{0}=N^{0} \longrightarrow$ $L^{2} \stackrel{\xi^{2}}{\longrightarrow} L^{3} \longrightarrow N^{3}$, the induced map of cohomology $\mu^{3}$ is equal to $\xi^{2}$. The remaining homomorphisms can be similarly verified.

Theorem 2.4. Given a les $\mathrm{L}$ of abelian groups there exists a ses of complexes of abelian groups (2.5) whose cohomology sequence is $\mathrm{L}$.

$$
\mathcal{O} \longrightarrow \mathcal{C} \longrightarrow \mathcal{D} \longrightarrow \mathcal{E} \longrightarrow \mathcal{O}
$$

Proof: Iterate the process of (2.2) until a commutative diagram of ses's (2.6) is produced

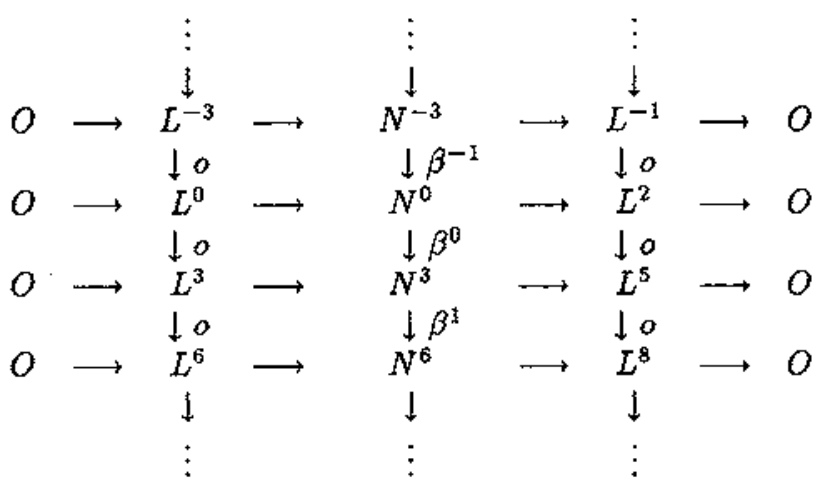

$\beta^{1} \beta^{0}=N^{0} \rightarrow K^{3} \longrightarrow N^{3} \rightarrow K^{6} \longrightarrow N^{6}=o$ from (2.1). Similarly $\beta^{i} \beta^{i-1}=0$ for all $i$, which verifes that the middle column is a complex. It is an immediate consequence of Lemma 2.3 that the cohomology of $(2.6)$ is $\mathrm{L}$.

Remarks 2.7. From (2.1) there are obvious configurations of groups and homomorphisms

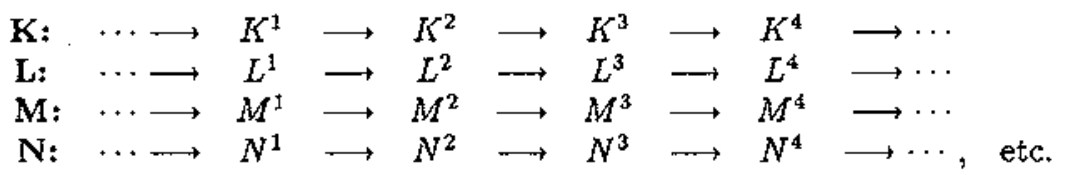


Each $K^{i} \longrightarrow K^{i+1}$ morphism is zero; each $L^{i} \longrightarrow L^{i+2}$ composite is zero; each $M^{i} \longrightarrow M^{i+3}$ composite is zero; and each $N^{i} \longrightarrow N^{i+4}$ composite is zero. The nomenclature 1-plex, 2-plex (complex), 3-plex (triplex), and 4-plex (quadriplex) lend themselves to this situation.

It is clear that given a homomorphism $\mathbf{N} \longrightarrow \mathbf{N}^{t}$, homomorphisms of the higher levels $(\mathbf{M}, \mathbf{L}, \mathbf{K})$ are induced. The converse is not true in general: a homomorphism of les's $\mathbf{L} \longrightarrow \mathbf{L}^{\prime}$ does not induce homomorphisms at the lower levels. This is a major obstruction to demonstrating a homomorphism of ses's of complexes which realizes a given homomorphism of les's.

Note that by simply reindexing $L$, other ses's of complexes can be found whose cohomology les is $\mathrm{L}$. The use of "cohomology" could be replaced by "homology" throughout by changing notation and reindexing $L^{k}$ by $L_{-k}$.

\section{Realization of les's from ses's of complexes of free abelian groups}

In the previous section, it was proven that any les $L$ is the cohomology les of a ses of complexes of abelian groups. In Algebraic Topology, it is frequently the case that complexes of free abelian groups are utilized to obtain les's. This section is devoted to the demonstration of the fact that it is also the case here that $\mathrm{L}$ is the les of a ses of complexes of free abelian groups.

The usefulness of general theorems in category theory will be shown here through their use in the elimination of 4- dimensional diagram chasing. If $\mathcal{S} E S$ (respectively $\mathcal{F} S E S$ ) denotes the category of ses's of complexes of abelian (respectively free abelian) groups, and if $\mathcal{L} A B$ denotes the category of les's of abelian groups, then the resuit to date is that

$$
H^{*}: \mathcal{S E S} \longrightarrow \mathcal{L} A B
$$

is onto for objects. It will be shown here that

$$
H^{*}: \mathcal{F} S E S \longrightarrow \mathcal{L} A B
$$

is also onto. It would be of interest to know whether $H^{*}$ is full (Remarks 2.7) e.g. can every morphism of $\mathcal{L} A B$ be represented as $H^{*}(g)$ for some homomorphism of ses's of complexes of abelian groups.

Lemma 3.1. Given any complex of abelian groups $\mathcal{B}$, there is an acyclic (exact) complex of free abelian groups which maps onto $B$.

Proof: Choose free abelian groups $F^{i}$ with epimorphisms $f^{i}: F^{i} \rightarrow B^{i}$, for each value of $i$. Denote by $e^{i}$ and $g^{i}$ the morphisms

$$
e^{i}=[0,1: 0,0]: F^{i-1} \oplus F^{i} \longrightarrow F^{i} \oplus F^{i+1},
$$


and

$$
g^{i}=\left\langle d^{i-1} f^{i-1}, f^{i}>: F^{i-1} \oplus F^{i} \longrightarrow B^{i}\right.
$$

These give rise to an epinorphism of complexes from the exact complex $\mathcal{F}$ to $\mathcal{B}, g: \mathcal{F} \longrightarrow \mathcal{B}$, which gives the desired mapping.

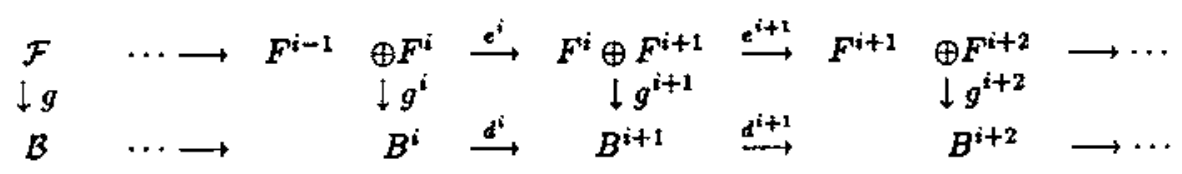

The kernel $\mathcal{N}$ of $g$ is a complex of free abelian groups. The ses of complexes $\mathcal{O} \longrightarrow \mathcal{N} \longrightarrow \mathcal{F} \stackrel{g}{\longrightarrow} \mathcal{B} \longrightarrow \mathcal{O}$ gives rise to a les of cohomology

$$
\cdots \rightarrow H_{0}^{n}(\mathcal{F}) \longrightarrow H^{n}(\mathcal{B}) \stackrel{\cong}{\longrightarrow} H^{n+1}(\mathcal{N}) \longrightarrow H_{0}^{n+1}(\mathcal{F}) \longrightarrow \cdots
$$

$\mathcal{F}$ is called a carefree cover of $\mathcal{B}$ to suggest that it is a carefully chosen free acyclic cover. This gives a means of realizing the cohomology of $\mathcal{B}$ by a free abelian complex $\mathcal{N}$.

The mapping cone construction on the identity map of a complex $B$ forms a new complex $\mathcal{M} B$ where $[\mathcal{M} B]^{i}=B^{i+1} \oplus B^{i}$ and $[\delta]^{i}=\left[-d^{i+1}, 0:-1, d^{i}\right]$. It is easily verified that $\mathcal{M} B$ is acyclic and that $\{0,1\}: B^{i} \longrightarrow B^{i+1} \oplus B^{i}$ and $<1,0>: B^{i+1} \oplus B^{i} \longrightarrow B^{i+1}$ induce the morphisms for a ses of complexes

$$
\mathcal{O} \longrightarrow \mathcal{B} \leftrightarrows \mathcal{L} B \stackrel{\pi}{\longrightarrow} \Sigma \mathcal{B} \longrightarrow \mathcal{O} \text {. }
$$

This process is functorial! Given the ses of complexes (2.5), there is a commutative diagram of ses's of complexes (3.2).

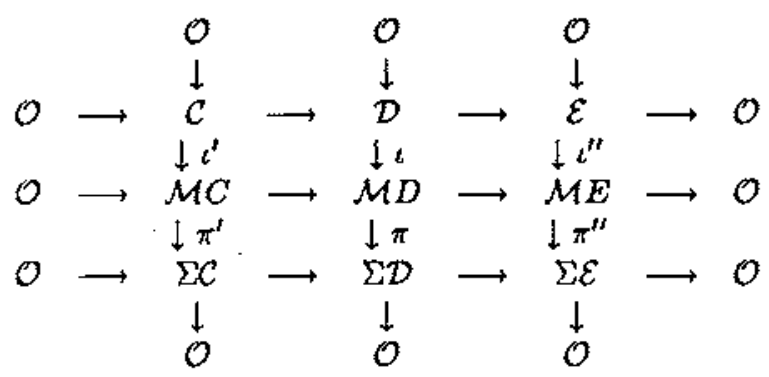

To avoid 4-dimensional diagram chasing in the course of checking that a mapping of a free short exact sequence of complexes to $\mathcal{O} \longrightarrow \mathcal{C} \longrightarrow \mathcal{D} \longrightarrow$ $\mathcal{E} \longrightarrow \mathcal{O}$ is commutative, the following argument is used. Pick a carefree cover $k: \mathcal{F}^{\prime} \rightarrow \mathcal{M C}$ and compose this as required to get $p=\pi^{\prime} k: \mathcal{F}^{\prime} \rightarrow$ $\Sigma \mathcal{C}, \ell: \mathcal{F}^{\prime} \longrightarrow \mathcal{M D}$, and $q=\pi \ell: \mathcal{F}^{\prime} \longrightarrow \Sigma \mathcal{D}$. Pick a second carefree 
cover $m: \mathcal{F}^{\prime \prime} \rightarrow \mathcal{M D}$ and use the morphisms of (3.2) to extend this to covers $r=\pi m: \mathcal{F}^{\prime \prime} \longrightarrow \Sigma \mathcal{D}, n: \mathcal{F}^{\prime \prime} \rightarrow \mathcal{M} E$, and $s=\pi^{\prime \prime} n: \mathcal{F}^{\prime \prime} \longrightarrow \Sigma \mathcal{E}$. There is a commutative diagram of complexes

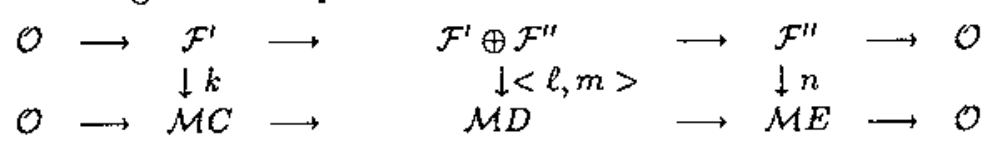

This gives rise to a ses in $\mathcal{C A B}^{2} \circ \longrightarrow k \longrightarrow<\ell, m>\longrightarrow n \longrightarrow$.

The homomorphisms $p, q, r, s$ give a free acyclic cover of the ses of complexes $\mathcal{O} \longrightarrow \Sigma \mathcal{C} \longrightarrow \Sigma \mathcal{D} \longrightarrow \Sigma \mathcal{E} \longrightarrow \mathcal{O}$ which leads to the commutative diagram (3.4) in $\mathcal{C} A B$.
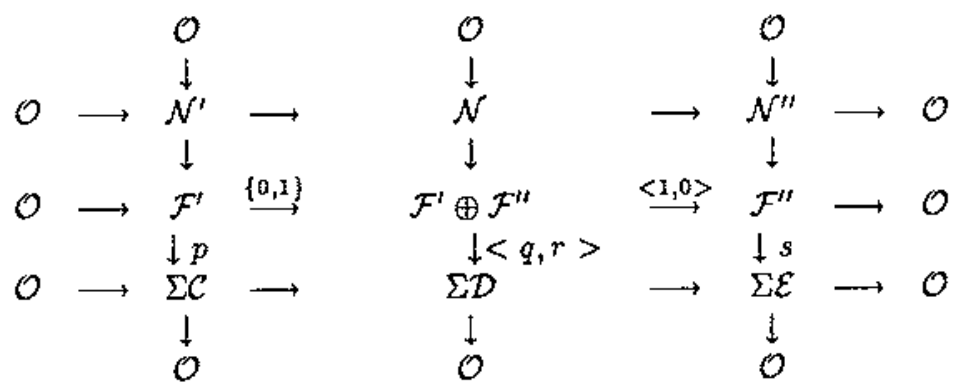

The ses of complexes of free abelian groups

$$
\mathcal{O} \longrightarrow \mathcal{N}^{\prime} \longrightarrow \mathcal{N} \longrightarrow \mathcal{N}^{\prime \prime} \longrightarrow \mathcal{O}
$$

has the correct cohomology because, since the middle sow of (3.4) is acyclic, the cohomology of (3.4) becomes

$$
\begin{aligned}
& \cdots H^{m-1}(\Sigma \mathcal{C}) \rightarrow H^{m-1}(\Sigma \mathcal{D}) \rightarrow H^{m-1}(\Sigma \mathcal{E}) \rightarrow H^{m}(\Sigma \mathcal{C}) \\
& \cong \downarrow \quad \cong 1 \quad \cong 1 \quad(-1) \quad \cong \\
& \cdots H^{m}\left(\mathcal{N}^{\prime}\right) \rightarrow H^{m}(\mathcal{N}) \quad \rightarrow \quad H^{m}\left(\mathcal{N}^{\prime \prime}\right) \quad \rightarrow \quad H^{m+1}\left(\mathcal{N}^{\prime}\right) \quad \ldots
\end{aligned}
$$

whereas that of $(3.2)$ is

$$
\begin{aligned}
& \cdots H^{m-1}(\Sigma \mathcal{C}) \rightarrow H^{m-1}(\Sigma \mathcal{D}) \rightarrow H^{m-1}(\Sigma \mathcal{E}) \rightarrow H^{m}(\Sigma \mathcal{C})
\end{aligned}
$$

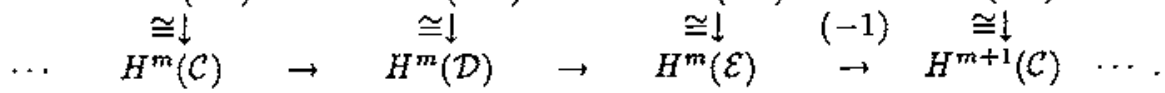

The squares marked with (-1) anticommute. Gluing (3.6) upside down onto (3.7) gives the required isomorphism of the cohomology of (3.5) with that of (2.5) and with the original les $\mathrm{L}$.

$$
\begin{aligned}
& \cdots \rightarrow H^{m}\left(\mathcal{N}^{\prime}\right) \rightarrow H^{m}(\mathcal{N}) \rightarrow H^{m}\left(\mathcal{N}^{\prime \prime}\right) \rightarrow H^{m+1}\left(\mathcal{N}^{\prime}\right) \rightarrow \cdots \\
& \cong \downarrow \quad \cong \downarrow \quad \cong \downarrow \\
& \cdots \rightarrow H^{m}(\mathcal{C}) \rightarrow H^{m}(\mathcal{D}) \rightarrow H^{m}(\mathcal{E}) \quad \rightarrow \quad H^{m+1}(\mathcal{C}) \rightarrow \cdots
\end{aligned}
$$

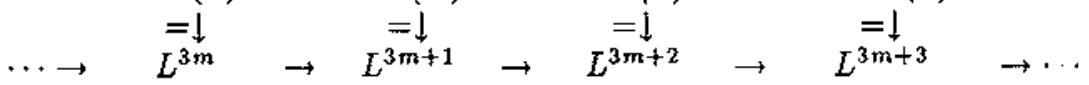


To construct the actual homomorphism of (3.5) to (2.5), note that (3.4) and (3.2) have identical bottom Iines, and that a homomorphism of the middle line of (3.4) to the middle line of (3.2) has been defined in (3.3). Working in the abelian category of morphisms of complexes of abelian groups $\mathcal{C} \mathcal{A B}{ }^{2}$ there is a commutative diagram (3.8) of morphisms which expresses this relationship between the diagram of complexes (3.3) and the identity morphisms.

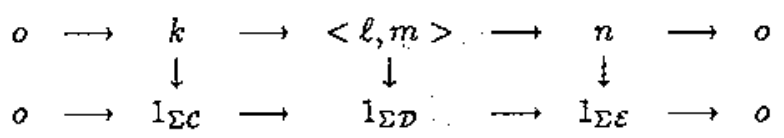

Since $\mathcal{C} A B^{2}$ is abelian, there is a unique, up to isomorphism, ses of kernels $o \longrightarrow \alpha \longrightarrow \beta \longrightarrow \gamma \longrightarrow o$ of the vertical epimorphisms in (3.8) where $\alpha, \beta$ and $\gamma$ are homomorphisms of complexes

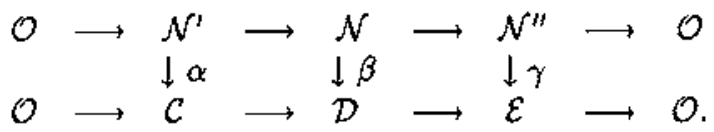

This gives the desired isonorphisms of cohomology, which proves the following theorem.

Theorem 3.10. Given a les L of abelian groups, there is a ses of complexes of free abelian groups whose cohomology les is $\mathrm{L}$.

The existence of this realization of $\mathbf{L}$ leads naturally to interesting questions of a topological nature. For example, if $L^{j}=O$ for all $j>0$ and for all $j<-k(k>0)$, and if all remaining $L^{j}$ are finitely generated abelian groups, then can $L$ be realized as the homology of the chain complex of a simplicial complex $K$ and a subcomplex $K_{0}$ ?

\title{
References
}

1. P.J. Hilton and I.S. Pressman, On completing Bicartesian Squares, Proc. Symp. Pure. Math. XVII, Appl. of Categorical Algebra (1970), 37-49.

2. P.J. Hilton and V. Stammbach, "A Course in Homological Algebra," Springer, New York-Heidelberg-Berlin, 1970.

3. S. MACLANE, "Homology," Springer, Berlin-Göttingen- Heidelberg, 1963.

\author{
Department of Mathematics and Statistics \\ Carleton University \\ Ottawa, KIS 5B6 \\ CANADA
}

Rebut el 22 de Desembre de 1988 